\title{
The general features of Bianchi-I cosmological models in Lovelock gravity
}

\author{
S.A. Pavluchenko \\ Special Astrophysical Observatory, Russian Academy of Sciences, Nizhnij Arkhyz, 369167 Russia
}

\begin{abstract}
We derived equations of motion corresponding to Bianchi-I cosmological models in the Lovelock gravity. Equations derived in the general case, without any specific ansatz for any number of spatial dimensions and any order of the Lovelock correction. We also analyzed the equations of motion solely taking into account the highest-order correction and described the drastic difference between the cases with odd and even numbers of spatial dimensions. For power-law ansatz we derived conditions for Kasner and generalized Milne regimes for the model considered. Finally, we discuss the possible influence of matter in the form of perfect fluid on the solutions obtained.
\end{abstract}

PACS numbers: 04.20.Fy, 04.50.-h, 04.50.Kd, 98.80.-k

\section{INTRODUCTION}

The idea that our space-time has more than four dimensions is not new in science. And since its first introduction by Kaluza [1] and Klein [2] it went a long way with different inspirations. Nowadays, the interest to such theories is mostly motivated by the superstrings and supergravity theories (see, e.g., [3]). The presence of extra dimensions makes it possible for superstrings theory to exist, but it leaves us with a gap between the number of dimensions the superstrings exist in, and our observable four-dimensional space-time. One of the possible ways to solve this problem is to make additional dimensions very small on cosmological scales by means of the cosmological theory.

It is well-known [4 [6] that the Einstein tensor is, in any dimension, the only symmetric and conserved tensor depending only on the metric and its first and second derivatives (with a linear dependence on second derivatives). If one drops the condition of linear dependence on second derivatives, one can obtain the most general tensor which satisfies other mentioned conditions - Lovelock tensor [7]. The corresponding Lagrangian (Lovelock Lagrangian), which generalizes the Einstein-Hilbert one, can be obtained by the same way - the Euler variation with respect to the metric. The Lovelock tensor differs from the Einstein tensor only if space-time has more than four dimensions, so the Lovelock gravity can be considered as a natural generalization of General Relativity in a higher-dimensional space-time. It contains quadratic and higher-order corrections in the Lagrangian, depending on the number of spatial dimensions.

In the cosmological context, the Lovelock gravity has as well been intensively studied (see, e.g., [8-12]). In our paper we study the Bianchi-I-type cosmological model in Lovelock gravity. As we pointed out above, the anisotropic cosmological models can possibly solve the issue with small extra dimensions. But to construct a self-consistent model, freed from singularities and other obstacles, one needs to fully understand the dynamics of such a system.

We decided to investigate Bianchi-I cosmological models. These are models with a simplest geometry and they can be used to understand common features of the model considered. The case with most general kind of geometry is more interesting, of course, but is much more complicated as well. And knowing some features from the Bianchi-I model analysis could be a great asset while studying more general cases. Not to mention, exploring Bianchi-I models is profitable by itself - indeed, according to nowadays cosmological point of view, our Universe is spatially flat, so as Bianchi-I models are, while general case, which usually involves products of Lie algebras, is not.

As a part of the analysis we demonstrate the difference between the cases with odd and even numbers of spatial dimensions. In our previous paper dedicated to the numerical investigation of the Gauss-Bonnet cosmologies [13] we noticed that there is a clear difference in the dynamics of $(4+1)$ - and $(5+1)$-dimensional models. Now we demonstrate that this difference is generic for all orders of Lovelock correction and for any number of dimensions.

Finally we take the matter in form of the perfect fluid into analysis. This decision is also motivated by our previous studies. In [14] we discovered the existence of "special solution" for pressureless fluid in (4+1)-dimensional GaussBonnet cosmology; in this paper, among other things, we demonstrate that this is the common feature of models with even number of spatial dimensions.

The structure of a manuscript is as follows: first, we derive the Lagrangian for the model with only one highest possible $n$ taken into account as well as equations of motion. Then, we consider cases with even and odd numbers of spatial dimensions separately, demonstrate the difference in the structure of the equations and, as a consequence, a difference in their dynamics. In section IV we apply so-called power-law anzatz to our equations and obtain conditions for Kasner and generalized Milne regimes; conditions obtained generalize known (for low $n$ ) ones. Then we take into account matter in form of a perfect fluid and investigate the influence of it onto the dynamics of the system. In Discussion we discuss the topics relevant to the results obtained and finally in the Conclusion sum up all the results. 


\section{EQUATIONS OF MOTION}

Lovelock invariants have the form [7]

$$
L_{n}=\frac{1}{2^{n}} \delta_{j_{1} j_{2} \ldots j_{2 n}}^{i_{1} i_{2} \ldots i_{2 n}} R_{i_{1} i_{2}}^{j_{1} j_{2}} \ldots R_{i_{2 n-1} i_{2 n}}^{j_{2 n-1} j_{2 n}},
$$

where $\delta_{j_{1} j_{2} \ldots j_{2 n}}^{i_{1} i_{2} \ldots i_{2 n}}$ is the generalized Kronecker delta of the order $2 n$. The Lagrangian density have a form

$$
\mathcal{L}=\sqrt{-g} \sum_{n} \alpha_{n} L_{n}
$$

where $g$ is the determinant of metric tensor, $\alpha_{n}$ is a constant of the order of Planck length in $n$ dimensions and summation over all $n$ in consideration is assumed. In our analysis we take into account only one highest possible correction - we are interested in the behavior in the vicinity of the cosmological singularity. In that regime higher curvature corrections are dominating over lower ones so one can consider highest possible correction only. In that case (2) contains only one term with highest possible $n$ :

$$
\mathcal{L}=\sqrt{-g} L_{n}
$$

since we have only one $n$, we can renormalize $L_{n}$ to set $\alpha_{n}$ to unity. As we mentioned in Introduction, we work with Bianchi-I-type metric in $(D+1)$-dimensional space-time:

$$
d s^{2}=\operatorname{diag}\left(-1, a_{1}^{2}(t), a_{2}^{2}(t), \ldots a_{D}^{2}(t)\right)
$$

where $a_{i}(t)$ is a scale factor, corresponding to $i$-th spatial dimension. From this metric one can build the following non-zero Riemann tensor components:

$$
R_{0 i 0 i}=-a_{i}(t) \ddot{a}_{i}(t), \quad R_{i j i j}=a_{i}(t) \dot{a}_{i}(t) a_{j}(t) \dot{a}_{j}(t),
$$

where Latin indices correspond to the spatial coordinates, the dot above corresponds to a derivation with respect to time and standard relations between indices for Riemann tensor are kept in mind (see, e.g., [15]). By raising indices in Eq. (4) one can get

$$
R_{0 i}^{0 i}=\frac{\ddot{a}_{i}(t)}{a_{i}(t)}, \quad R_{i j}^{i j}=\frac{\dot{a}_{i}(t) \dot{a}_{j}(t)}{a_{i}(t) a_{j}(t)} .
$$

Since we are working in a flat space-time, it is convenient to rewrite Eq. (5) in terms of the Hubble parameter $H_{i} \equiv \dot{a}_{i}(t) / a_{i}(t)$ :

$$
R_{0 i}^{0 i}=\dot{H}_{i}+H_{i}^{2}, \quad R_{i j}^{i j}=H_{i} H_{j}
$$

Now we can substitute (6) into (1), imply the properties of the generalized Kronecker delta and obtain the expression for $n$-th order Lovelock invariant for Bianchi-I-type metrics:

$$
\begin{aligned}
L_{n}^{D}= & 2^{-n}\left((2 n-3) ! ! \sum_{i=1}^{D}\left[\left(\dot{H}_{i}+H_{i}^{2}\right) \sum_{\substack{\left\{j_{1}, j_{2}, \ldots j_{2 n-2}\right\} \neq i \\
j_{1}>j_{2}>\ldots j_{2 n-2}}} H_{j_{1}} H_{j_{2}} \ldots H_{j_{2 n-2}}\right]+\right. \\
& \left.+(2 n-1) ! ! \sum_{k_{1}>k_{2}>\ldots k_{2 n}} H_{k_{1}} H_{k_{2}} \ldots H_{k_{2 n}}\right)
\end{aligned}
$$

where $(2 n-1)$ !! is bifactorial of $(2 n-1)$ : $(2 n-1) ! !=(2 n-1) \times(2 n-3) \times \cdots$. There $D$ is a number of spatial dimensions and $D \geqslant 2 n$. Since we deal with only one (highest possible) $n$, we can neglect the $1 / 2^{n}$ multiplier as well as cancel $(2 n-3)$ !! with a reduction of $(2 n-1)$ !! and Eq. (7) become 


$$
\begin{aligned}
L_{n}^{D} & =\sum_{i=1}^{D}\left[\left(\dot{H}_{i}+H_{i}^{2}\right) \sum_{\substack{\left\{j_{1}, j_{2}, \ldots j_{2 n-2}\right\} \neq i \\
j_{1}>j_{2}>\ldots j_{2 n-2}}} H_{j_{1}} H_{j_{2}} \ldots H_{j_{2 n-2}}\right. \\
& +(2 n-1) \sum_{k_{1}>k_{2}>\ldots k_{2 n}} H_{k_{1}} H_{k_{2}} \ldots H_{k_{2 n}} .
\end{aligned}
$$

The first term in Eqs. (17) and (8) comes from the products of a $R_{0 i}^{0 i} R_{j k}^{j k} \ldots$ kind and the second one - from the products like $R_{i j}^{i j} R_{k l}^{k l} \ldots$. Since the same index can not appear twice, the products of a $R_{0 i}^{0 i} R_{0 j}^{0 j} \ldots$ kind are impossible.

Then one can substitute Eq. (8) into Eq. (3) and infer the equations of motion via varying; thus, the constraint equation takes a form

$$
(2 n-1) \sum_{k_{1}>k_{2}>\ldots k_{2 n}} H_{k_{1}} H_{k_{2}} \ldots H_{k_{2 n}}=0
$$

the dynamical equation corresponding to $i$-th spatial coordinate becomes

$$
\left.\begin{array}{rl}
\sum_{\substack{m=1 \\
m \neq i}}^{D} & \left.\left[\dot{H}_{m}+H_{m}^{2}\right) \sum_{\substack{\left\{j_{1}, j_{2}, \ldots j_{2 n-2}\right\} \neq\{i, m\} \\
j_{1}>j_{2}>\ldots j_{2 n-2}}} H_{j_{1}} H_{j_{2}} \ldots H_{j_{2 n-2}}\right]+ \\
+(2 n-1) & \sum_{\substack{\left\{k_{1}, k_{2}, \ldots k_{2 n}\right\} \neq i \\
k_{1}>k_{2}>\ldots k_{2 n}}}^{H_{k_{1}} H_{k_{2}} \ldots H_{k_{2 n}}}=0 .
\end{array}\right]+
$$

\section{THE DIFFERENCE BETWEEN THE $D=2 n$ AND $D=2 n+1$ CASES}

Eqs. (9) and (10) might look complicated but in fact they are quite simple; to illustrate it let us rewrite them for odd and even numbers of spatial dimensions. For this one can notice, that any given $n$ gives a maximal-order correction for an odd $D=2 n+1$ and even $D=2 n$ number of spatial dimensions. So for odd $(D=2 n+1)$ number of spatial dimensions, the constraint equation takes a form

$$
\sum_{k_{1}>k_{2}>\ldots k_{2 n}} H_{k_{1}} H_{k_{2}} \ldots H_{k_{2 n}}=0
$$

this is just the sum of all possible multiplications put in order with $2 n$ multipliers constructed from Hubble parameters. One can see there are only $(2 n+1)$ terms in this sum, and we omitted the $(2 n+1)$ multiplier in front of sum in (9) since it is always non-zero. The dynamical equation corresponding to $i$-th spatial coordinate become

$$
\left.\begin{array}{rl}
\sum_{\substack{m=1 \\
m \neq i}}^{D} & {\left[\left(\dot{H}_{m}+H_{m}^{2}\right) \sum_{\substack{\left\{j_{1}, j_{2}, \ldots j_{2 n-2}\right\} \neq\{i, m\} \\
j_{1}>j_{2}>\ldots j_{2 n-2}}} H_{j_{1}} H_{j_{2}} \ldots H_{j_{2 n-2}}\right.}
\end{array}\right]+
$$

For the case considered, the second term in Eq. (10) has only one possible combination - indeed, we have $(2 n+1)$ spatial dimensions, one of them is "forbidden" (since this equation corresponds to the $i$-th coordinate) and the remaining $2 n$ coordinates have only one multiplication combination with $2 n$ multipliers.

For an even number of spatial dimensions $(D=2 n)$ we have a constraint equation in the following form:

$$
H_{k_{1}} H_{k_{2}} \ldots H_{k_{2 n}}=0
$$

The same with the Eq. (11) reasoning, we have only one multiplication with $2 n$ multipliers constructed from Hubble parameters for this case. And this multiplication is equal to zero. This leaves us the first drastic difference between 
the cases with odd and even numbers of spatial dimensions - the latter should have one of Hubble parameters always equal to zero. The dynamical equations for the odd-number case:

$$
\sum_{\substack{m=1 \\ m \neq i}}^{D}\left(\dot{H}_{m}+H_{m}^{2}\right) H_{j_{1}} H_{j_{2}} \ldots H_{j_{2 n-2}}=0 .
$$

One can clearly see the difference between Eqs. (10) and (12): the last term vanished due to the absence of multiplications of this type and the r.h.s. of the first term is a multiplication with $2 n-2$ terms: one index is "forbidden" because it's an $i$-th dynamical equation, another one comes into $\left(\dot{H}_{m}+H_{m}^{2}\right)$ part, consequently we are left exactly with $2 n-2$ "free" Hubble parameters which form only one put in order multiplication with $2 n-2$ multipliers.

Therefore one can clearly see the difference between the cases with odd and even number of spatial dimensions they differ both in the constraint equation as well as in dynamical ones. In [14] (and previously in [13]) we showed the difference between the $(4+1)$ and $(5+1)$ Gauss-Bonnet-dominated $\left(L_{2}\right)$ Universe; now we see that this difference is global and that it is applied to all orders of Lovelock gravity.

\section{POWER-LAW ANZATZ}

Since the Kasner's paper [16], the power-law anzatz for Bianchi-I model is widely used for various reasons. It implies the scale factors to be written as $a_{i}(t)=t^{p_{i}}$, so the Hubble parameters would be $H_{i}=p_{i} / t$ and the derivation of Hubble parameters with respect to time would have the form $\dot{H}_{i}=-p_{i} / t^{2}$. Let us rewrite the equations of motion using this anzatz and obtain some important results. Thus, constraint equation of our system takes a form

$$
\sum_{k_{1}>k_{2}>\ldots k_{2 n}} p_{k_{1}} p_{k_{2}} \ldots p_{k_{2 n}}=0
$$

we omit the $(2 n-1) / t^{2 n}$ multiplier since only one $n$ (the highest possible) is taken into account and we deal with a vacuum case. The dynamical equations become (again, we write down only one $i$-th equation)

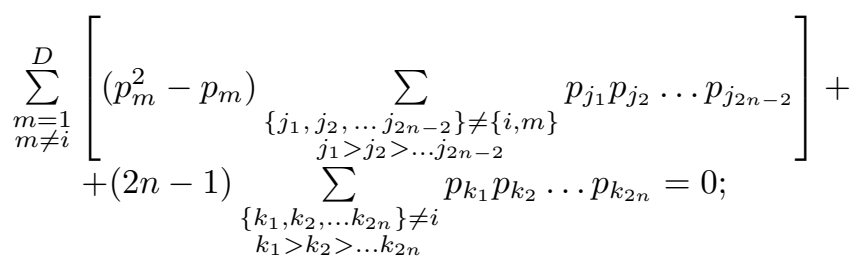

and we omit the $t^{2 p_{m}-2 n}$ multiplier. Needless to say, all the discussion in the previous section about the difference between the cases with odd and even numbers of spatial dimensions is valid for this anzatz, and the analogs of Eqs. (11) 14) could be easily written.

Now let us find the conditions for the analogs of Kasner regime. In finding them we followed [17]. Let us consider the sum of all dynamical equations - the sum of all Eqs. (16) for all $i$. We will have a sum

$$
\begin{aligned}
& \sum_{[i]} \sum_{\substack{m=1 \\
m \neq i}}^{D}\left[\left(p_{m}^{2}-p_{m}\right) \sum_{\substack{\left\{j_{1}, j_{2}, \ldots j \\
j_{1}>j_{2}>\ldots j_{2 n-2}\right\} \neq\{i, m\}}} p_{j_{1}} p_{j_{2}} \ldots p_{j_{2 n-2}}\right]+ \\
& \quad+(2 n-1) \sum_{\substack {[i] \\
\begin{subarray}{c}{\left\{k_{1}, k_{2}, \ldots k_{2 n}\right\} \neq i \\
k_{1}>k_{2}>\ldots k_{2 n}{ [ i ] \\
\begin{subarray} { c } { \{ k _ { 1 } , k _ { 2 } , \ldots k _ { 2 n } \} \neq i \\
k _ { 1 } > k _ { 2 } > \ldots k _ { 2 n } } }\end{subarray}} p_{k_{1} p_{k_{2}} \ldots p_{k_{2 n}}}=0 .
\end{aligned}
$$

Taking (15) into account, one can see that the second term in (17) is zero. As for the first term, it can be decomposed into two parts - with only linear multipliers and with only quadratic ones:

$$
\begin{aligned}
& (D+1-2 n) \sum_{i=1}^{D} p_{i}^{2} \sum_{\substack{\left\{j_{1}, j_{2}, \ldots j_{2 n-2}\right\} \neq i \\
j_{1}>j_{2}>\ldots j_{2 n-2}}} p_{j_{1}} p_{j_{2}} \ldots p_{j_{2 n-2}}- \\
& -(2 n-1)(D+1-2 n) \sum_{i>j_{1}>j_{2}>\ldots j_{2 n-2}} p_{i} p_{j_{1}} p_{j_{2}} \ldots p_{j_{2 n-2}}=0
\end{aligned}
$$


the coefficients before the terms are the result of simple combinatorics. Cancelling $(D+1-2 n)$ one would obtain a relation, which depends only on the order of Lovelock correction and not on the number of spatial dimensions:

$$
\sum_{i=1}^{D} p_{i}^{2} \sum_{\substack{\left\{j_{1}, j_{2}, \ldots j_{2 n-2}\right\} \neq i \\ j_{1}>j_{2}>\ldots j_{2 n-2}}} p_{j_{1}} p_{j_{2}} \ldots p_{j_{2 n-2}}-(2 n-1) \sum_{j_{1}>j_{2}>\ldots j_{2 n-1}} p_{j_{1}} p_{j_{2}} \ldots p_{j_{2 n-1}}=0
$$

Now let's consider a sum

$$
\sum_{i=1}^{D} p_{i} \sum_{j_{1}>j_{2}>\ldots j_{2 n-1}} p_{j_{1}} p_{j_{2}} \ldots p_{j_{2 n-1}}
$$

This sum falls into the terms of two kinds - if $i=\left\{j_{1}, j_{2}, \ldots j_{2 n-1}\right\}$, its terms are like the first term in (18), if $i \neq\left\{j_{1}, j_{2}, \ldots j_{2 n-1}\right\}-$ like the second ones. After some simple combinatorics, one can rewrite this sum as

$$
\begin{aligned}
& \sum_{i=1}^{D} p_{i} \sum_{j_{1}>j_{2}>\ldots j_{2 n-1}} p_{j_{1}} p_{j_{2}} \ldots p_{j_{2 n-1}}=\sum_{i=1}^{D} p_{i}^{2} \sum_{\substack{\left\{j_{1}, j_{2}, \ldots j_{2 n-2}\right\} \neq i \\
j_{1}>j_{2}>\ldots j_{2 n-2}}} p_{j_{1}} p_{j_{2}} \ldots p_{j_{2 n-2}}+ \\
& +2 n \sum_{i>j_{1}>j_{2}>\ldots j_{2 n}} p_{i} p_{j_{1}} \ldots p_{j_{2 n}} .
\end{aligned}
$$

From (15) it is obvious that the second term is equal to zero. So finally

$$
\sum_{i=1}^{D} p_{i} \sum_{j_{1}>j_{2}>\ldots j_{2 n-1}} p_{j_{1}} p_{j_{2}} \ldots p_{j_{2 n-1}}=\sum_{i=1}^{D} p_{i}^{2} \sum_{\substack{\left\{j_{1}, j_{2}, \ldots j_{2 n-2}\right\} \neq i \\ j_{1}>j_{2}>\ldots j_{2 n-2}}} p_{j_{1}} p_{j_{2}} \ldots p_{j_{2 n-2}}
$$

Combining (18) and (19) results in

$$
\sum_{i=1}^{D} p_{i} \sum_{j_{1}>j_{2}>\ldots j_{2 n-1}} p_{j_{1}} p_{j_{2}} \ldots p_{j_{2 n-1}}=(2 n-1) \sum_{j_{1}>j_{2}>\ldots j_{2 n-1}} p_{j_{1}} p_{j_{2}} \ldots p_{j_{2 n-1}}
$$

so either

$$
\sum_{i=1}^{D} p_{i}=(2 n-1)
$$

or

$$
\sum_{j_{1}>j_{2}>\ldots j_{2 n-1}} p_{j_{1}} p_{j_{2}} \ldots p_{j_{2 n-1}}=0
$$

First of the two is the condition for the analog of Kasner regime in $(D+1)$-dimensional Universe with Lovelock gravity of the order $n$, while the second corresponds to the generalized Milne solution (see the Discussion for more details).

\section{INFLUENCE OF MATTER}

In this section we will investigate the influence the matter in form of a perfect fluid exerts upon the dynamics of the system. The equations of motion in this case take the form 


$$
G_{\mu \nu}=\frac{8 \pi G_{D}}{3} T_{\mu \nu}
$$

where $G_{D}$ is a $D$-dimensional gravitation constant, $T_{\mu \nu}$ is the stress-energy tensor of a perfect fluid

$$
T_{\mu \nu}=(\rho+p) u_{\mu} u_{\nu}-p g_{\mu \nu} \quad \text { with an equation of state } p=w \rho,
$$

and $G_{\mu \nu}$ is a generalization of the Einstein tensor in Lovelock gravity (Lovelock tensor; for issues about its properties as a generalization of Einstein tensor, see, e.g., [18]):

$$
G_{\mu \nu}=\frac{\delta \mathcal{L}}{\delta g^{\mu \nu}}-\frac{1}{2} g_{\mu \nu} \mathcal{L} \quad \text { where } \mathcal{L} \text { is the Lagrangian (3). }
$$

So, the equations of motion would take a form:

for constraint

$$
(2 n-1) \sum_{k_{1}>k_{2}>\ldots k_{2 n}} H_{k_{1}} H_{k_{2}} \ldots H_{k_{2 n}}=\rho
$$

and for dynamical equations (again, $i$-th equation)

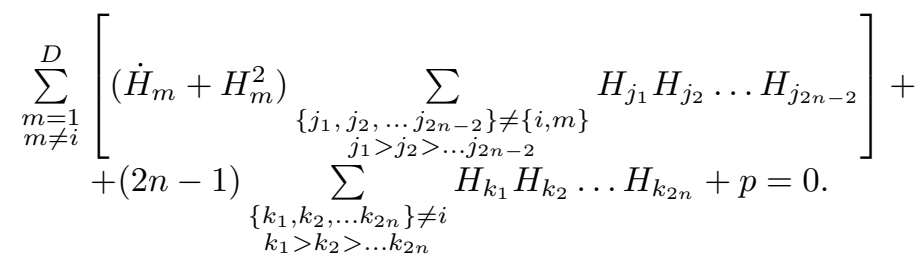

In the equations (25, (26)), we renormalized the density $\rho$ in order to avoid the $8 \pi G_{D} / 3$ term - we are interested in the qualitative description so we can do this. And of course, in addition to (25, 26) one needs a continuity equation:

$$
\dot{\rho}+(\rho+p) \sum_{i=1}^{D} H_{i}=0 .
$$

The system (25) 27), along with initial conditions, completely determines the dynamics of the system and its past and future evolution.

Let us rewrite (25) 26) for the power-law anzatz. It's easy to see that Lovelock tensor scales as

$$
\begin{aligned}
& G_{00}=(2 n-1) t^{-2 n} \sum_{1} p_{i}, \\
& G_{j j}=t^{2 p_{j}-2 n} \sum_{2} p_{i},
\end{aligned}
$$

where $\sum_{1} p_{i}$ and $\sum_{2} p_{i}$ are the corresponding sums in l.h.s. of Eqs. (15) and (16), respectively (one can easily verify that both terms in Eq. (16) scale the same). Solving (27) in the chosen anzatz, one can write down the scaling of the stress-energy tensor components:

$$
\begin{aligned}
& T_{00}=\rho_{0} t^{-(1+w) \sum p_{i}}, \\
& T_{j j}=w \rho_{0} t^{2 p_{j}-(1+w) \sum p_{i}} .
\end{aligned}
$$

Matching (28) and (29), one can find the condition under which system can be solved:

$$
\sum_{i=1}^{D} p_{i}=\frac{2 n}{1+w} .
$$

Finally, matching (20) with (30), one can find the "critical" value for the equation of state, with separate Lovelock and matter-dominating regimes (see the Discussion for more details):

$$
w_{c r}=\frac{1}{2 n-1} .
$$




\section{DISCUSSION}

We have derived the vacuum equations of motion (9) 10) for Bianchi-I-type metric for any number of spatial dimensions $(D \geqslant 4)$ in the Lovelock gravity of any order $n \geqslant 2$. The corresponding Lagrangian for $L_{2}$ was first derived by Lanczos [19] and for $L_{3}$ in [8]. One can verify, that for the chosen metric our result coincides with the previously obtained ones.

If one considers only the leading (in $1 / t$ order) terms, then one needs to take into consideration only the highestorder Lovelock contribution, it would be $n=[D / 2]$. Therefore we rewrote our equations of motion for the cases of odd $D=2 n+1$ (11, 12) and even $D=2 n$ (13, 14) numbers of spatial dimensions. One can easily see the difference between the two: while there are no restrictions from constraint on Hubble functions in case of odd numbers [of spatial dimensions] (Eq. (11)), the even-numbers case (Eq. (13)) sets a restriction: one of Hubble parameters should be always equal to zero. The dynamical equations also differ in structure (12) vs. (14)). Our numerical investigation of $(4+1)$ and $(5+1)$ cases in $L_{2}[14]$ proved that there is a significant difference between two cases. We believe the difference in $L_{2}$ is due to the mentioned above reasons, so one should expect similar features from higher-dimension cases as well.

When it comes to the anisotropic cosmological models in Lovelock gravity, two main cases are considered: product spaces and Bianchi-I models. As we claimed in Introduction, we consider the latter one. And for Bianchi-I-type models most of results are obtained in power-law anzatz. We also applied this anzatz to our solution and investigated it a bit. It has a form (15, 16) and coincides with previously found results for corresponding $n$ (in addition to the previously mentioned papers, let us add [20] with a result for $\left.\left[D=9, L_{4}\right]\right)$.

For the power-law anzatz we also found an expansion rate in terms of Kasner exponents: $\sum_{i} p_{i}=(2 n-1)$. This result also coincides with those previously reported for low $n$. Finally, we found the condition on $p_{i}$, which corresponds to the generalized Milne solution. It is determined from two conditions - (15) and (21). And here we can again see the difference between the cases with odd and even numbers of spatial dimensions. Indeed, for $D=2 n$, the Eq. (15) reduces to only one term directly implying that one of $p_{i}$ should be zero. With this, Eq. (21) also reduces to only one term implying another $p_{i}$ should also be zero; the rest of $p_{i}$ are arbitrary. Thus, the resulting $p_{i}$ layout is as follows: $\left(a_{1}, a_{2}, \ldots, a_{D-2}, 0,0\right)$. For $D=2 n+1$, the situation is a bit different - since $D=2 n+1$, we have $D$ terms in Eq. (15), and nullifying one of $p_{i}$ will leave us with only one term in l.h.s. of Eq. (15), and this eventually nullifies another $p_{i}$. But now with two of $p_{i}$ set to zero, the l.h.s. of Eq. (21) is also left with only one term and this sets third of $p_{i}$ to zero. Therefore for odd numbers of spatial dimensions the resulting $p_{i}$ layout is as follows: $\left(a_{1}, a_{2}, \ldots, a_{D-3}\right.$, 0, 0, 0). Finally, in a $D$-independent form: $\left(a_{1}, a_{2}, \ldots, a_{2 n-2}, 0, \ldots\right)$. A proof that there are no solutions except the two mentioned above (Kasner-like with (20) and generalized Milne with (21)), is quite similar to the proof for $L_{2}^{5}$ [13].

Finally we studied possible influence of matter in the form of perfect fluid on the dynamics of the system. We wrote the equations of motion (25]27) and deepened the difference between the cases with odd and even numbers of spatial dimensions. In the presence of matter, even-dimensional cases change drastically - without the matter one of Hubble functions should be zero (see Eq. (13) ), while even a small amount of matter changes the situation - in the presence of matter $(\rho \neq 0)$ none of Hubble parameters can be zero (see Eq. (25) ).

We as well found a condition for the system to be solvable in the power-law anzatz (Eq. (30)). Matching (20) and (30), one can find a "critical" value for the equation of state, with separates Lovelock and matter-dominating regimes. In [14] we demonstrated that this value separates two different asymptotic [leading to initial singularity] regimes if $w>w_{c r}$ the initial singularity is isotropic (and the matter terms are dominating), and if $w<w_{c r}$ the initial singularity is anisotropic (and the Gauss-Bonnet terms are dominating). We believe that the same situation holds in the higher orders of Lovelock gravity, though some numerical simulations might be an asset. Finally, if one rewrite Eqs. (25) and (26) for $D=2 n$ and $w=0$ in terms of $p_{i}$, they would become $p_{k_{1}} p_{k_{2}} \ldots p_{k_{2 n}}=\rho$ for the constraint and

$$
\sum_{\substack{m=1 \\ m \neq i}}^{D} p_{m}\left(p_{m}-1\right) p_{j_{1}} p_{j_{2}} \ldots p_{j_{2 n-2}}=0
$$

for the dynamic equations (there we omit the powers of $t$ ). One can see, that the "special solution" $p_{i} \equiv 1$, which was described in [14] for $L_{2}^{4}$, is valid for the general case of Lovelock gravity in even numbers of spatial dimensions.

And the last, but nonetheless a very important note. All through the present paper we claim that our results are obtained with only one highest-order Lovelock correction taken into account. In reality, some of them can be generalized for a mixture of Lovelock corrections with different $n$. If one wants to deal with some different Lovelock corrections at once (this would be the case if one wants to study the dynamics not only in the vicinity of an initial singularity), one should use (2) instead of (3) as a Lagrangian density and substitute all individual $L_{n}$ needed into it. But in this case one need to use (7) expression for $L_{n}$ since we cancel $2^{-n} \times(2 n-3)$ !! terms in (8) in order to 
simplify it for the case where only one $n$ is used. Now if we combine this $n$-dependent multiplier with $\alpha_{n}$ into new constant $\tilde{\alpha}_{n}$, equations take a form

$$
\sum_{i} \tilde{\alpha}_{i} P_{i}=0
$$

where $P_{i}$ are the equations of motion corresponding to $L_{i}-$ l.h.s. of Eq. (9) for a constraint or Eq. (10) for a dynamical equation. Thus, equations can be easily constructed from the already derived ones. The same comes for the matterfilled universe, but in this case in the r.h.s. of (32) would be $\rho$ for a constraint or $(-p)$ for the dynamical equations (compare with (25) and (26)).

From the way we derived the equations for the power-law anzatz one can see that the power-law analogs of equations cannot be generalized for the mixture of $n$. This comes out from the way we derived them - as you remember, we omitted the powers of $t$ since they were the only ones for vacuum case or the same with those in r.h.s. for the matter case. But with a mixture of $n$ we would have different powers of $t$ for different $n$ and so we cannot cancel them anymore. With this, the results obtained from the power-law anzatz (like different sums of $p_{i}$ ), cannot be applied for the case with mixture of $n$. Yet, the results for the highest possible $n$ can be used as an asymptotic regime for this case.

\section{CONCLUSIONS}

In this paper we investigated Bianchi-I cosmological models in Lovelock gravity. We were first to derive the Lagrangian and the equations of motion for the model considered: Bianchi-I model in Lovelock gravity with only highest possible correction taken into account. This by itself is a great achievement - previously, when one deals with Bianchi-I models in Lovelock gravity, only one specific order was involved. This is linked with a difficulty in obtaining equations from the most general case. In our paper we imply symmetries of Bianchi-I model to obtain our results; our result is correct only for Bianci-I case but it cover all possible $n$ as well as is valid for any number of spatial dimensions.

We analyzed obtained equations of motion separately for the cases of odd and even number of spatial dimensions and proved that there is a difference between these two cases.

After applying power-law anzatz to our equations of motion we were able to obtain conditions for the Kasner as well as generalized Milne regimes. Results obtained matches those for low- $n$ cases and generalize them.

Finally, we took the matter in form of perfect fluid into consideration. For this case we also obtained equations of motion; condition this equations can be resolved under and the "critical" value for the equation of state which separates Lovelock-dominating from matter-dominating regimes. Results of the analysis of the model with matter make the mentioned above difference between $D=2 n$ and $D=2 n+1$ cases even deeper.

We also discussed the way of constructing the equations of motion for a mixture of $n$ from the obtained equations both for vacuum and for matter cases.

All results obtained matches with existing results for low- $n$ cases and generalize them for all possible $n$.

\section{Acknowledgments}

I would like to thank A.V. Toporensky and I.V. Kirnos for a stimulating discussion and S.V. Karpov for technical help.

[1] T. Kaluza, Sit. Preuss. Akad. Wiss. K1, 966 (1921).

[2] O. Klein, Z. Phys. 37, 895 (1926).

[3] M. Green, J. Schwarz, and E. Witten, Superstrings (Cambridge University Press, Cambridge, England, 1987).

[4] H. Vermeil, Nachr. Ges. Wiss. Göttingen (Math.-Phys. Klasse, 1917) p. 334 (1917).

[5] H. Weyl, Raum, Zeit, Materie, 4th ed. (Springer, Berlin, 1921).

[6] E. Cartan, J. Math. Pure Appl. 1, 141 (1922).

[7] D. Lovelock, J. Math. Phys. 12, 498 (1971).

[8] F. Müller-Hoissen, Phys. Lett. 163B, 106 (1985).

[9] J. Madore, Phys. Lett. 111A, 283 (1985); J. Madore, Class. Quant. Grav. 3, 361 (1986); F. Müller-Hoissen, Class. Quant. Grav. 3, 665 (1986). 
[10] N. Deruelle, Nucl. Phys. B327, 253 (1989).

[11] T. Verwimp, Class. Quant. Grav. 6, 1655 (1989); G. A. Mena Marugán, Phys. Rev. D 42, 2607 (1990); ibid. 46, 4340 (1992).

[12] N. Deruelle and L. Fariña-Busto, Phys. Rev. D 41, 3696 (1990).

[13] S.A. Pavluchenko and A.V. Toporensky, Mod. Phys. Lett. A 24, 513 (2009) // arXiv:0811.0558.

[14] I.V. Kirinos, A.N. Makarenko, S.A. Pavluchenko, and A.V. Toporensky, arXiv:0906.0140.

[15] S. Weinberg, Gravitation and Cosmology (Wiley, New York, 1972);

L. D. Landau and E. M. Lifshitz, The Classical Theory of Fields (Pergamon Press, 4th Edition, Oxford, 2002).

[16] E. Kasner, Am. Journ. Math., 43, 217 (1921).

[17] A. Toporensky, and P. Tretyakov, Grav. Cosmol. 13, 207 (2007) // arXiv:0705.1346

[18] M. Farhoudi, Gen. Rel. Grav. 41, 117 (2009).

[19] C. Lanczos, Z. Phys. 73, 147 (1932); C. Lanczos, Ann. Math. 39, 842 (1938).

[20] J. Demaret et al., Phys. Rev. D 41, 1163 (1990).

[21] B. Zumino, Phys. Rep. 137, 109 (1986). 